\title{
Intestinal anastomotic injury alters spatially defined microbiome composition and function
}

\author{
Benjamin D Shogan' ${ }^{1}$, Daniel P Smith ${ }^{2,4}$, Scott Christley ${ }^{1}$, Jack A Gilbert ${ }^{2,3}$, Olga Zaborina ${ }^{1}$ and John C Alverdy ${ }^{1 *}$
}

\begin{abstract}
Background: When diseased intestine (i.e., from colon cancer, diverticulitis) requires resection, its reconnection (termed anastomosis) can be complicated by non-healing of the newly joined intestine resulting in spillage of intestinal contents into the abdominal cavity (termed anastomotic leakage). While it is suspected that the intestinal microbiota have the capacity to both accelerate and complicate anastomotic healing, the associated genotypes and functions have not been characterized.

Results: Using 165 rRNA amplicon sequencing of samples collected on the day of surgery (postoperative day 0 (POD0)) and the 6th day following surgery (postoperative day 0 (POD6)), we analyzed the changes in luminal versus tissue-associated microbiota at anastomotic sites created in the colon of rats. Results indicated that anastomotic injury induced significant changes in the anastomotic tissue-associated microbiota with minimal differences in the luminal microbiota. The most striking difference was a 500-fold and 200-fold increase in the relative abundance of Enterococcus and Escherichia/Shigella, respectively. Functional profiling predicted the predominance of bacterial virulence-associated pathways in post-anastomotic tissues, including production of hemolysin, cytolethal toxins, fimbriae, invasins, cytotoxic necrotizing factors, and coccolysin.
\end{abstract}

Conclusion: Taken together, our results suggest that compositional and functional changes accompany anastomotic tissues and may potentially accelerate or complicate anastomotic healing.

Keywords: Colon anastomosis, $16 \mathrm{~S}$ rRNA, PiCRUST, Bacterial composition, Predicted function, Anastomotic tissues, Luminal content

\section{Background}

Removal (resection) of a segment of the gastrointestinal tract is a common procedure performed by general surgeons for a variety of illnesses including cancer, diverticulitis, or intestinal obstruction. When surgeons resect a diseased segment of the gastrointestinal tract, intestinal continuity is reestablished by carefully suturing or stapling the remaining ends together to create a viable and well-sealed connection referred to as an 'anastomosis'. An anastomosis therefore is a significant tissue injury given that the surgeon must first divide all of the blood vessels feeding the proposed resection site; all layers of the intestinal wall are divided meaning that their entire thickness from mucosa to serosa is disrupted and then tissues are approximated with sutures and

\footnotetext{
*Correspondence: jalverdy@surgery.bsd.uchicago.edu

'Department of Surgery, University of Chicago, 5841 S. Maryland, Chicago, IL 60637, USA

Full list of author information is available at the end of the article
}

staples that represent foreign bodies embedded into the area of healing tissues. As such, surgeons routinely administer antibiotics directed against the intestinal microbiota to decontaminate the intestine of its microflora in an effort to reduce the presumed effect of the microbiota on healing [1]. Despite excellent suture technique by highly skilled surgeons to approximate the tissues and fuse and seal the intestinal wall, without proper subsequent healing of the anastomotic tissues, 'leak' can occur resulting in spillage of intraluminal contents into the peritoneal cavity that is associated with sepsis, permanent need for an ostomy, and death. Thus, despite excellent surgical technique, leaks still occur and its pathogenesis remains unknown. While surgeons have known for over 60 years that the intestinal microbiota play a key and critical role in the etiopathogensis of leakage [2], the species involved and the molecular details of their activity remain

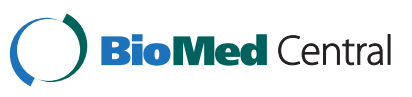


unknown. Work from our lab has recently confirmed that both the species and phenotype of microbes present on anastomotic tissues is a critical determinant of anastomotic leakage following intestinal resection and anastomosis [3]. In this study, we identified that when high swarming phenotypes of Pseudomonas aeruginosa are present on anastomotic tissues, they are associated with leaks. Yet, the choice of antibiotics to provide prophylaxis to patients is based on culture-derived results of the luminal microbiota before and after antibiotic administration that are now several decades old. Because anastomotic tissues are generally inaccessible, it is extremely difficult to track changes in microbial content over the course of healing. As a result, it is not known whether the community structure, membership, and/or function of the luminal microbiota are representative of the microbiota that associate with anastomotic tissues themselves. Furthermore, it is now recognized that culture-based studies of the luminal microbiota fail to classify the majority of the microbial diversity present. Finally, genetic sequencing has revealed the dependency of intestinal microbial community structure, membership, and function on the regional and spatial context in which they establish niche specialization $[4,5]$. Therefore, there is a significant gap in knowledge as to whether healing anastomotic tissues harbor a distinct microbial community from the luminal microbial community. Elucidation of this issue may have a significant impact on the rationale for choosing antibiotics in preparation for colon surgery.

Therefore, the aims of this study were to create anastomotic injury in rats similar to that which is performed in humans and to determine the changes and differences in the luminal versus tissue microbiota over a 6-day course of anastomotic healing.

\section{Results}

Anastomosis induces changes in the composition of the intestinal microbiota associated with intestinal tissue, but does not affect the microbiota associated with luminal contents

Rats were subjected to removal (resection) of $1 \mathrm{~cm}$ of colon just above the colorectal junction followed by reconnection (anastomosis) as is typically performed in humans with colon cancer of the distal intestine. DNA was isolated from resected colon and colon luminal content at the time of surgery, represented as postoperative day 0 (POD0). On postoperative day 6 (POD6), rats were sacrificed and the luminal and tissue associated microbiota at the anastomotic segment were harvested for analysis. Results demonstrated that colon surgery did not affect the microbial community structure associated with the luminal contents (stool), but induced significant changes in the composition of the intestinal microbiota associated with the intestinal tissue (Figure 1A,B).

\section{The tissue microbiota at POD6 is enriched by commensal opportunistic bacteria}

The taxonomic profiling of tissue-associated microbiota demonstrated that most of the changes in bacterial composition were observed in the phyla Proteobacteria, Actinobacteria, and Firmicutes (Figure 2). Among Proteobacteria, the relative abundance of Escherichia/Shigella (c_Gammaproteobacteria;o_Enterobacteriales;f_Enterobacteriaceae) increased 200-fold. In Actinobacteria, the relative abundance of an uncultured bacterium (c_Coriobacteria;o_Coriobacteriales;f_Coriobacteriaceae) increased approximately 50-fold (Figure 3). Non-uniform changes among the genera Firmicutes were observed. The relative abundance of uncultured bacteria representing Ruminococcaceae and Clostridia decreased 20 -fold, while the relative abundance of
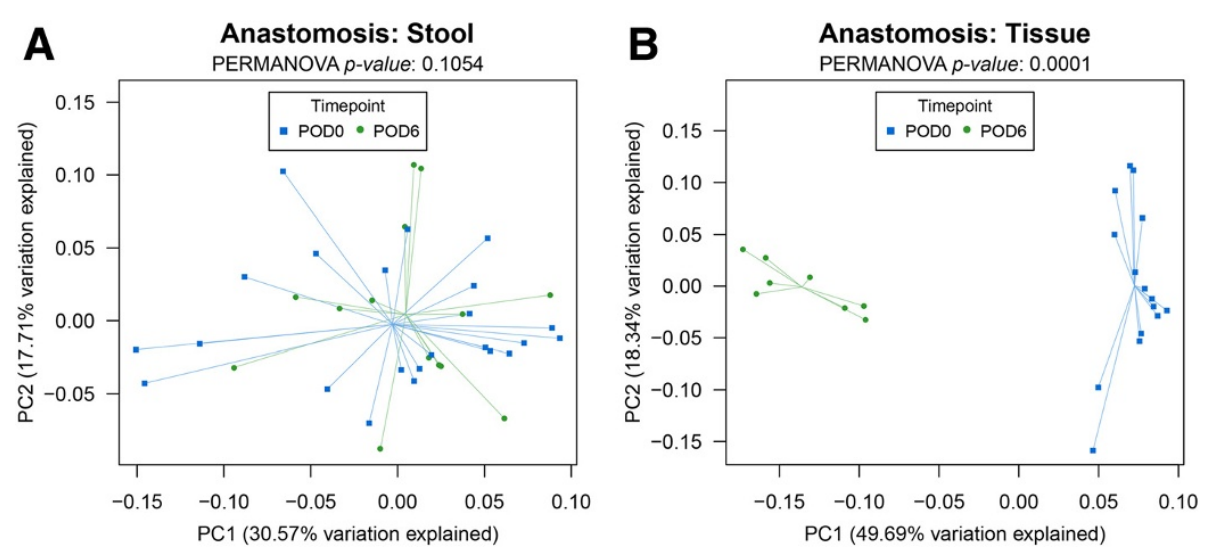

Figure 1 Principal components analysis of luminal- and tissue-associated microbiomes. Colon surgery affects the composition of the intestinal microbiota associated with intestinal tissue (B) but not the microbiota associated with luminal contents (A). $n=10$ per group. PERMANOVA $p$ values based on 10,000 random permutations of the dataset. Multiple comparisons were performed using the Bonferroni method. Error bars $= \pm 2$ standard error. 

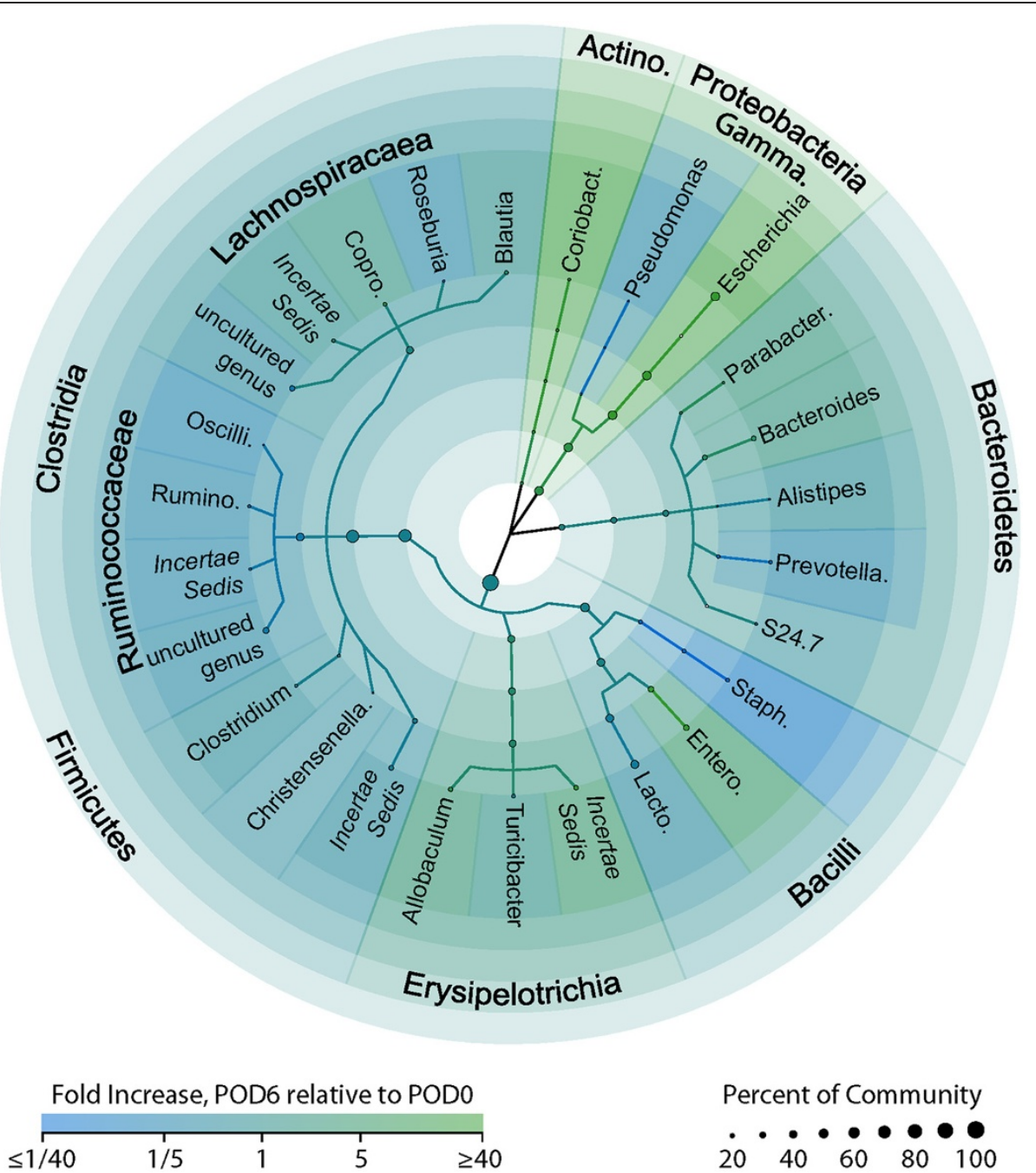

Figure 2 Taxonomic tree of anastomotic tissue microbiome changes. Shading indicates increases (green) and decreases (blue) in relative abundances of taxonomic groups on POD6 relative to PODO. Point sizes represent the higher point-in-time average abundance of the group relative to the entire microbial community. Groups comprising less than 1\% of the total community on POD0 and POD6 are excluded.

the genera Allobaculum and Coprococcus increased approximately 10-fold, and Enterococcus increased 500-fold (Figure 3). Finally, a 20-fold decrease in the relative abundance of Prevotellaceae, demonstrated that Bacteroidetes were also influenced by anastomotic injury (Figure 3).

\section{Predicted functional profiling suggests the potential of virulence-associated pathways in post-anastomotic tissues}

The functional potential of the microbiota associated with the intraluminal contents (stool) and tissue before (POD0) and after anastomosis (POD6) was predicted using PiCRUST [6]. There were no statistically significant functional differences for the intraluminal contents between POD0 and POD6, while the microbiota associated with intestinal tissue revealed multiple significantly altered functional attributes after anastomosis (Table 1).
The upregulated functions in tissue-associated microbiota can be traits of Escherichia and Enterococcus. The virulence-associated functions such as pore formation by hemolysin E [7], bacterial attachment to mammalian cells via AIDA-I adhesin-like protein [8], cytotoxicity to eukaryotic cells by cytolethal toxins [9] and cytotoxic necrotizing factor 1 [10], increased motility (outer membrane usher protein, fimbrial-like protein, type 1 fimbrial protein, fimbrial chaperone protein) [11], and invasion of epithelial cells via invasion B [12] can be attributed to Enterobacteriaceae, particularly, Escherichia. The proteolytic cleavage by coccolysin also known as gelatinase can be attributed to Enterococcus [13,14]. Importantly, gelatinase GelE of Enterococcus faecalis has been shown to degrade collagen, a critical component for anastomotic tissue healing $[15,16]$. We further performed enrichment analysis for KEGG metabolic pathways to determine 


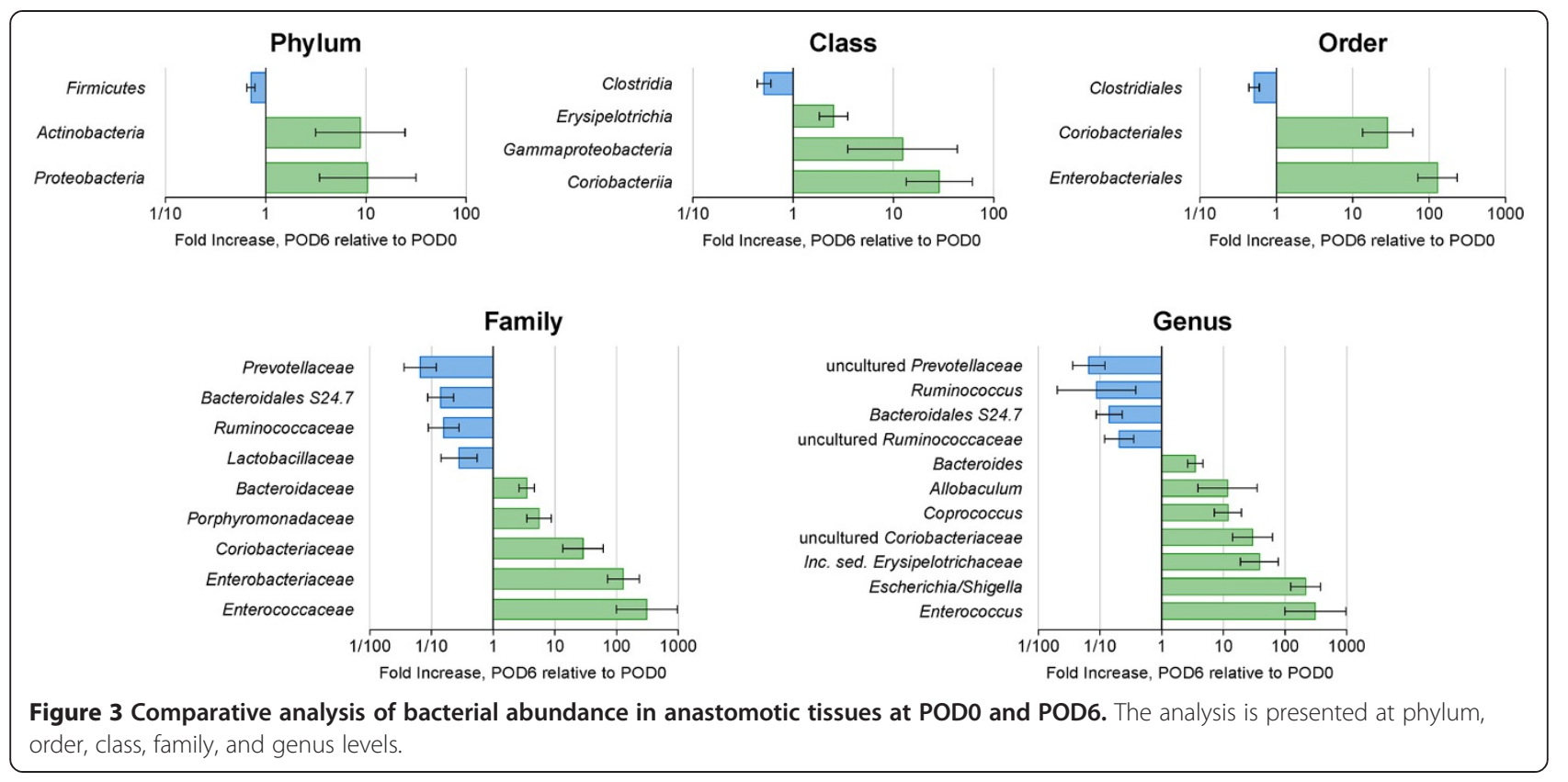

what metabolic changes occur at post-anastomotic tissues versus stool. The analysis indicates there are substantially more metabolic changes for tissue-associated microbiota than for the microbiota in the luminal contents, as expected based on changes in microbial community structure (Figure 4). Furthermore, the analysis of post-anastomotic tissues predicts virulence-associated metabolic pathways such as lipopolysaccharide biosynthesis, bacterial secretion system, and shigellosis, which are not represented in the stool microbiota. The metabolic pathways were linked to the OTUs responsible for the functions (Figure 5). The analysis demonstrated a shift in the primary contributors to the metabolic functions such as genus Lactobacillus, order Clostridiales, and genus Ruminococcus for pre-anastomotic tissue versus family Enterobacteriaceae, genus Enterococcus, and genus Bacteroides for post-anastomotic tissue (Figure 5).

\section{Discussion}

Advances in non-culture-based analyses of the intestinal microbiome have demonstrated that environmental influences (e.g., diet, antibiotic therapy, age, colonization history, geography) have profound effects on microbiota community structure, membership, and function [18], which are region specific within the gastrointestinal tract $[4,5]$. Although intestinal surgery in the form of resection and anastomosis has been practiced for decades, until now, there has been no systematic assessment of the bacterial diversity and functional potential associated with this surgery. It has been observed in germ-free and antibiotic-treated animals that disruption of the normal microbiota results in a weaker intestinal anastomosis as measured by burst strength (i.e., the pressure at which an anastomosis will burst) $[19,20]$. In these studies, they also observed a loss of collagen, a major determinant of anastomotic healing and burst strength. Conversely, empiric antibiotic treatment in other animal models prevents anastomotic leak when pathogenic bacteria or pathologic conditions are present [21]. This latter observation suggests that tissue injury alone, and the physiologic stress it incurs, may significantly influence the microbiome.

Our results indicate that intestinal resection and anastomosis induce a change in the microbiota associated with intestinal tissues, which is not observed in the luminal contents. Our findings also predict that tissueassociated microbial communities express an adhesive phenotype, which may explain, in part, their tropism toward injured tissues. Previous work from our laboratory has indicated that soluble products released during surgical injury can act as both chemoattractants and 'cues' that induce a phenotype shift among pathogenic bacteria such as $P$. aeruginosa $[22,23]$. In the present study, anastomotic tissues may select for microbes that express enhanced virulence (i.e., E. faecalis) and thus may have the potential to complicate anastomotic healing. A critical loss of the cytoprotective microbiota (i.e., Firmicutes and Bacteroidetes) on anastomotic tissues may further facilitate colonization and invasion by disease-associated microbiota, again with the potential to complicate healing. The precise explanation by which the process of surgery in the present study shifted the intestinal microbiota remains to be clarified. The surgery performed in the present study included a large abdominal incision, colon resection, anastomosis, and wound closure, all of which has been shown to result in a catabolic stress and 
Table 1 Predicted upregulated functions in tissue-associated microbiota after anastomosis

\begin{tabular}{|c|c|c|c|c|}
\hline $\begin{array}{l}\text { KEGG } \\
\text { orthology }\end{array}$ & Description & Pathway/function & $\begin{array}{l}\text { Primary } \\
\text { contributor }\end{array}$ & $p$ value \\
\hline K03276 & $\begin{array}{l}\text { UDP-glucose/galactose:(glucosyl)LPS alpha-1, } \\
\text { 2-glucosyl/galactosyltransferase [EC:2.4.1.-] }\end{array}$ & Lipopolysaccharide biosynthesis & Enterobacteriaceae & 0.02 \\
\hline K11139 & Hemolysin E & Pore-forming toxin [7] & Enterobacteriaceae & 0.02 \\
\hline K03765 & Transcriptional activator of cad operon & $\begin{array}{l}\text { The cad operon encodes a system for } \\
\text { neutralization of low extracellular pH }\end{array}$ & Enterobacteriaceae & 0.02 \\
\hline K12678 & AIDA-I adhesin-like protein & $\begin{array}{l}\text { Autotransporter family porin, mediates bacterial } \\
\text { attachment to mammalian cells [8] }\end{array}$ & Enterobacteriaceae & 0.02 \\
\hline K09925 & Hypothetical protein & & & 0.02 \\
\hline K11014 & Cytolethal distending toxin subunit B (CdtB) & $\begin{array}{l}\text { Initiates a eukaryotic cell cycle block at the } \mathrm{G} 2 \\
\text { stage prior to mitosis, CdtB potentiates a } \\
\text { cascade leading to cell cycle block [9] }\end{array}$ & Enterobacteriaceae & 0.02 \\
\hline K11015 & Cytolethal distending toxin subunit $\mathrm{C}(\mathrm{CdtC})$ & $\begin{array}{c}\mathrm{CdtC} \text { and } \mathrm{CdtA} \text { function as dimeric subunits, } \\
\text { which bind } \mathrm{CdtB} \text { and delivers it to the mammalian } \\
\text { cell interior }\end{array}$ & Enterobacteriaceae & 0.02 \\
\hline K11013 & Cytolethal distending toxin subunit A (CdtA) & Same as above & Enterobacteriaceae & 0.02 \\
\hline K11264 & $\begin{array}{c}\text { Methylmalonyl-CoA decarboxylase } \\
\text { [EC:4.1.1.41] }\end{array}$ & Propanoate metabolism & Enterobacteriaceae & 0.02 \\
\hline K13244 & $\begin{array}{c}\text { c-di-GMP-specific phosphodiesterase } \\
\text { [EC:3.1.4.52] }\end{array}$ & Hydrolases acting on ester bonds & Enterobacteriaceae & 0.02 \\
\hline K03459 & Formate transporter (focB) & Electrochemical potential-driven transporters & Enterobacteriaceae & 0.02 \\
\hline K07354 & Outer membrane usher protein (sfmD) & Bacterial motility proteins [11] & Enterobacteriaceae & 0.02 \\
\hline K07355 & Fimbrial-like protein (sfmF) & Bacterial motility proteins & Enterobacteriaceae & 0.02 \\
\hline K07352 & Type 1 fimbrial protein (sfmA) & Bacterial motility proteins & Enterobacteriaceae & 0.02 \\
\hline K07353 & Fimbrial chaperone protein & Bacterial motility proteins & Enterobacteriaceae & 0.02 \\
\hline K12985 & $\begin{array}{c}\text { (Galactosyl)LPS 1,2-glucosyltransferase } \\
{[\text { EC:2.4.1.-] }}\end{array}$ & Lipopolysaccharide biosynthesis proteins & Enterobacteriaceae & 0.02 \\
\hline K10708 & $\begin{array}{l}\text { Fructoselysine 6-phosphate deglycase } \\
\text { [EC:3.5.--] }\end{array}$ & $\begin{array}{l}\text { Hydrolases acting on carbon-nitrogen bonds, other } \\
\text { than peptide bonds }\end{array}$ & Enterobacteriaceae & 0.02 \\
\hline K11008 & Cytotoxic necrotizing factor 1 (cnf1) & Blocks cell cycle G2/M transition in uroepithelial cells [10] & Enterobacteriaceae & 0.02 \\
\hline K11920 & AraC family transcriptional regulator (envY) & DNA-binding transcriptional regulator & Enterobacteriaceae & 0.02 \\
\hline K13285 & Invasin B & Bacterial invasion of epithelial cells $[12,17]$ & Enterobacteriaceae & 0.03 \\
\hline K08605 & Coccolysin [EC:3.4.24.30] (gelE) & Metallopeptidases $[13,14]$ & Enterococcus & 0.03 \\
\hline
\end{tabular}

immune activation as a compensatory mechanism to deal with the process of healing the wounds [24,25]. There likely exist multiple mechanisms that drive the compositional and functional changes in the microbiota including redistribution of nutrients away from the gut to healing tissues, immune activation, and changes in luminal and mucosal oxygen status [26]. It is also possible that the microbiota 'sense' a dramatic change in host health status and undergo compositional and functional changes as a mechanisms of adaptation until recovery to homeostasis is complete. Further work is needed to determine precisely which of the factors predominate in this model.

It is noteworthy that most of the current antibiotic regimens used as prophylaxis agents for intestinal surgery are based on culture-based analyses of expelled stool. The limitation of this approach is that antibiotics are not based on a tissue-specific microbiome. As such, we do not know which tissue microbiota are associated with healing versus non-healing. This may be critically important to prevent complications such as anastomotic leaks, which still persist today at unacceptable rates even under the most expert of care [27]. While antibiotic use has been demonstrated to be highly effective in preventing anastomotic leak, the type, duration, and microbial targets of currently recommended antibiotics have remained empirical and invalidated. Data from the present study would suggest that direct genomic and functional analyses of the tissue-associated microbiota at anastomotic sites may provide a more directed approach to antibiotic use in anastomotic surgery by understanding their effect on the microbial community structure, membership, and function.

The observed 500-fold increase in Enterococcus in postoperative anastomotic tissues is interesting. Although enterococci are commensal inhabitants of the mammal gut, 


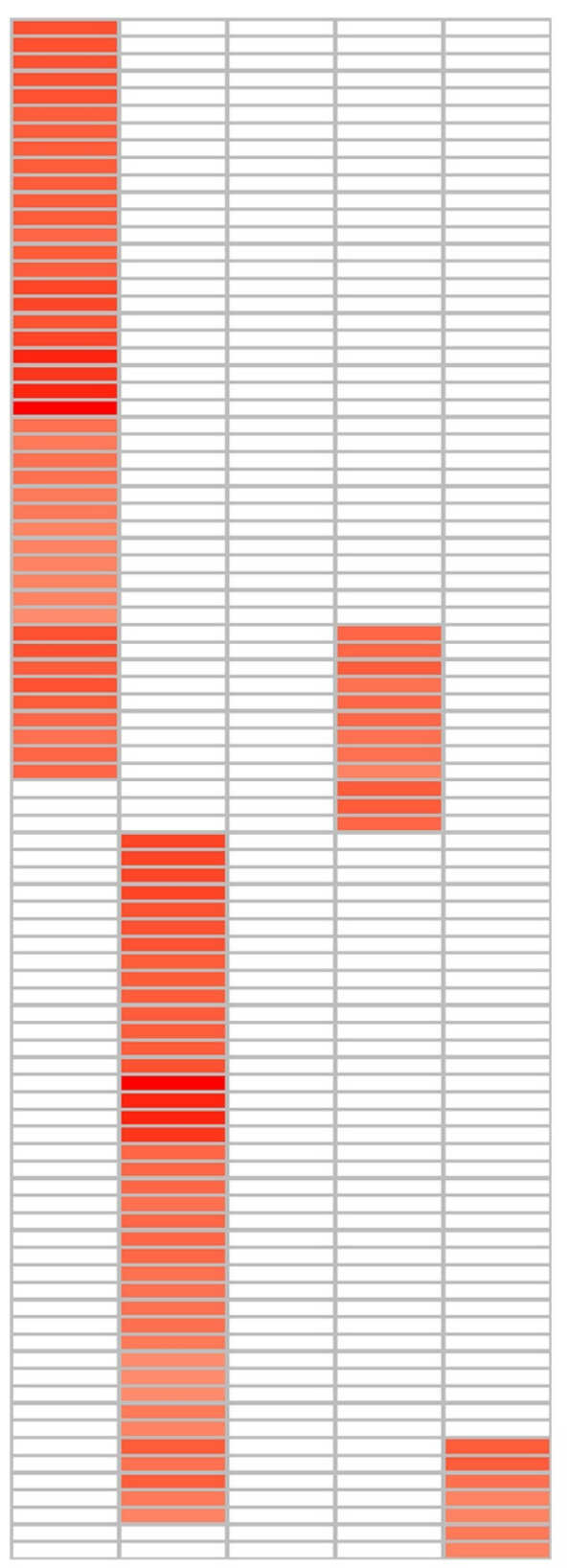

ko00061: Fatty acid biosynthesis
k000670: One carbon pool by folate

ko00670: One carbon pool by folate
ko00626: Naphthalene degradation

KOOB020: RNA polymerase

ko00730: Thiamine metabolism

ko00791: Atrazine degra

ko00471: D-Glutamine and D-glutarnate metabolism

kock010: Ribosorne

ko00900: Terpenoid backbone biosynthesis
ko00720: Carbon fixation pathways in prokaryotes

ko04112: Cell cycle - Caulobacter.

ko03420: Nucleotide excision repair

ko00561: Glycerolipid metabolism

kodo650. Butanoate metabolisin

ko00362: Benzoate degradation
k000083: Drug metabolism - other enzymes

k000473. D-Alanine metabolism

ko00290. Valine, leucine and isoleucine biosynthesis

ko00970: Aminoacyl-tRNA biosynthesis

ko02040: Flagellar assembly

$k 000642$ : Ethylbenzene degradation

ko02030. Bacterial chemotaxis

ko05145. Toxoplasmosis

ko05150: Staphylococcus aureus infection

ko03450: Non-homologous end-joining

KoC3030: DNA replication

ko04115: $\mathrm{D} 53$ signaling pathway

ko04626: Plant-pathogen interaction

ko00440: Phosphonate and phosphinate metabolism

KocB320: PPAR signaling pathway

(a)

ko000550: Capotidonold ycan biosynthesis

ko000300: Lysine biosynthesis

ko00621: Dioxin degradation

ko03440: Homnologous recombination

ko00062: xylene degradation

ko00620: Pyruvate metabolism
k000270: Cysteine and methionine metabolism

kodocas. Pentose phosphate pathway.

ko00010: Glycolysis / Gluconeogenesis
ko00710 Carbon fixation in photosynthetic organisms

(r)

ko00130. Ubigunone and other terpenoid-quinone biosynthesis

ko00051: Fructose and mannose metabolism

ko000312: beta-Lactam resistance

k00028. Gerahiol degradation

(1)

halosporin biosynthesis

ko00910: Nitrogen metabolism

ko00623: Toluene degradation

ko00250: Alanine, aspartate and glutarnate metabolism

k00033. Nitrotoluene degradation

ko00790: Folate biosynthesis

ko02060: Phosphotransferase system (PTS)

ko00531: Glycosaminoglycan
ko00780: Biotin metabolism

koo00\% Brotin metabolism

k000540: Lipopolysaccharide biosynthesis

ko00523. Polyketide sugar unit biosynthesis

ko01040: Bios ynthesis of unsaturated fatty acids

k000190: Oxicative phosphorylation

kocotion. bela-Alanine melabolism

koo 0005 . Glyoxylate and dicarboxylate metabolis

Kooc030: Ascorbate and aldarate

ko05111. Vibrio cholerae pathogenic cycle

ko05131: Shigellosis

k000260: Glycine, serine and threonine metabolism

ko000504: Glycerophospholipid metabolism

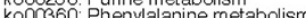

ko 03018: RNA degradation
ko00380: Tryptophan metabolism

k001053: Biosynthesis of siderophore group nonribosomal peptides

roo

kooutsolism

ko00140: Steroid harmone biosynthesis

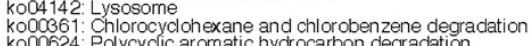

ko00624: Polycycic aromatic hydrocarbon degradation

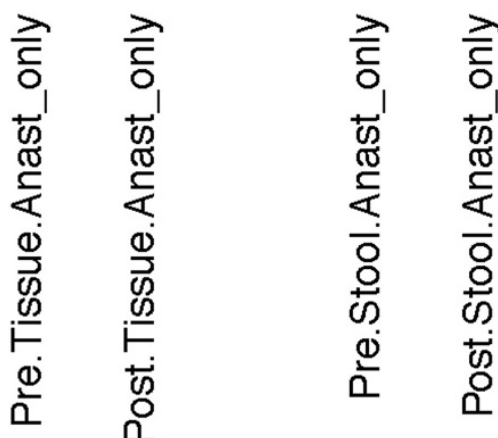

\section{Color Key}

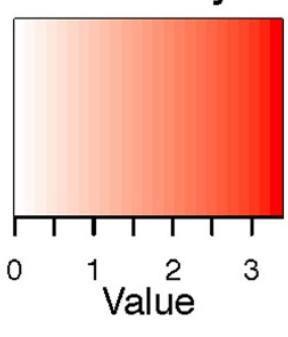

Figure 4 (See legend on next page.) 
(See figure on previous page.)

Figure 4 Enriched metabolic pathways for pre- and post-anastomic tissue and stool. Pathway enrichment for KEGG metabolic pathways using HUMAnN followed by statistical comparative analysis using LEfSe was performed to determine differential enrichment between POD0 and POD6 for tissue and stool. Red color indicates that the KEGG metabolic pathway is enriched in comparison to the corresponding POD for either tissue or stool. Tissue and stool were not directly compared with each other, so common metabolic pathways do not indicate equivalent levels of enrichment for both.

they have been observed to be regularly associated with human infections [28]. In terms of intestinal anastomoses, it has been reported that E. faecalis is highly prevalent at leaking anastomotic tissues, although its role as a causative agent in leakage remains unknown $[29,30]$. Enterococcus has a high adherence affinity to extracellular cellular matrix proteins such as fibronectin, laminin, and various different types of collagens [31-33] including collagen IV [34]. What role, if any, Enterococcus plays in the etiopathogenesis of anastomotic leak will require further study. However, the fact that its abundance is increased to such a high degree raises the possibility that it may complicate healing given that it is known to confer a high degree of pathogenicity when it predominates. In fact, PICRUSt analysis [6] in the current work predicted the capacity of anastomotic tissues-associated microflora to possess coccolysin known as a gelatinase GelE of E. faecalis that is able to degrade collagen. Similarly, Escherichia genus also displayed a dramatic increase in relative abundance (i.e., 200-fold) at anastomotic tissues which is another commensal capable of confer a pathogenic phenotype [35]. Further work will be necessary to independently assess the virulence potential of strains isolated from anastomotic tissues versus luminal contents in both cases.

Given that the normal microbiota provide pathogen colonization resistance [36-38], there may be value in understanding how the microbiota assemble from the lumen to anastomotic tissues over the course of anastomotic healing. For example, our results demonstrated that there is a postoperative decrease in Ruminococcaceae, a family of Firmicutes that belong to obligate anaerobes. It has been recently shown that the families Ruminococcaceae and Lachnospiraceae (Clostridium cluster XIV group) colonize mucosal folds in the proximal murine colon [5]. Taken together, our data indicate that a significant membership of the normal microbiota may be lost at anastomotic tissues, the consequence of which is unknown. The family Lactobacillaceae was also reduced at anastomotic tissues and its known cytoprotective action [39-41] to accelerate healing may play a role in leak when they are critically decreased and additional physiologic stress occurs.

Although taxonomic profiling by the $16 \mathrm{~S}$ rRNA analysis can be very informative, it does not provide any functional context to the bacterial communities. Metagenomics and metatranscriptomics would provide greater detail on their potential and actual functions yet are still technically challenging in terms of analysis. However, using PICRUSt [6], we observed predicted global changes in metabolic and virulence functions of bacterial communities among microbiota associated with anastomotic tissues. These findings provide a path forward in which we can apply this technique to models of anastomotic leak and determine its predictive capacity and its ability to inform a microbial mechanism for anastomotic leakage.

\section{Conclusion}

An anastomotic injury appears to significantly alter the tissue-associated microbiota without affecting the luminal microbiota. A more detailed analysis of the tissues associated microbiota over the full course of anastomotic healing could lead to novel strategies for a leak prevention that may include allowing for a balance of microbial community structure, membership, and function.

\section{Methods}

\section{Rat model of anastomosis}

The Institute for Animal Care and Use Committee at the University of Chicago approved all animal experiments. Adult, male Wistar rats 250-300 g (Charles River Laboratory, Chicago, IL, USA) were used for all experiments and were allowed unrestricted access to rat chow and tap water. Prior to surgery, rats were sedated with $40-80 \mathrm{mg} / \mathrm{kg}$ ketamine, 5-10 mg/kg xylazine via an intraperitoneal injection. Using aseptic technique, rats were subjected to an abdominal incision (laparotomy) and a $0.5-\mathrm{cm}$ distal colon resection followed by an endto-end rectosigmoid reconnection (anastomosis) using 12 interrupted 6-0 non-absorbable sutures. Colon luminal content was collected for DNA isolation. The resected piece of colon was washed with saline and then swabbed for tissue associated microbiota. In all cases, anastomotic integrity was confirmed immediately after construction by injecting saline into the rectum and observing that the anastomotic was water tight. On postoperative day 6 all rats were sacrificed and the luminal contents adjacent to anastomosis were collected. For tissue-associated microbiota, anastomotic tissues were removed, and mechanically homogenized, and then DNA was extracted. 


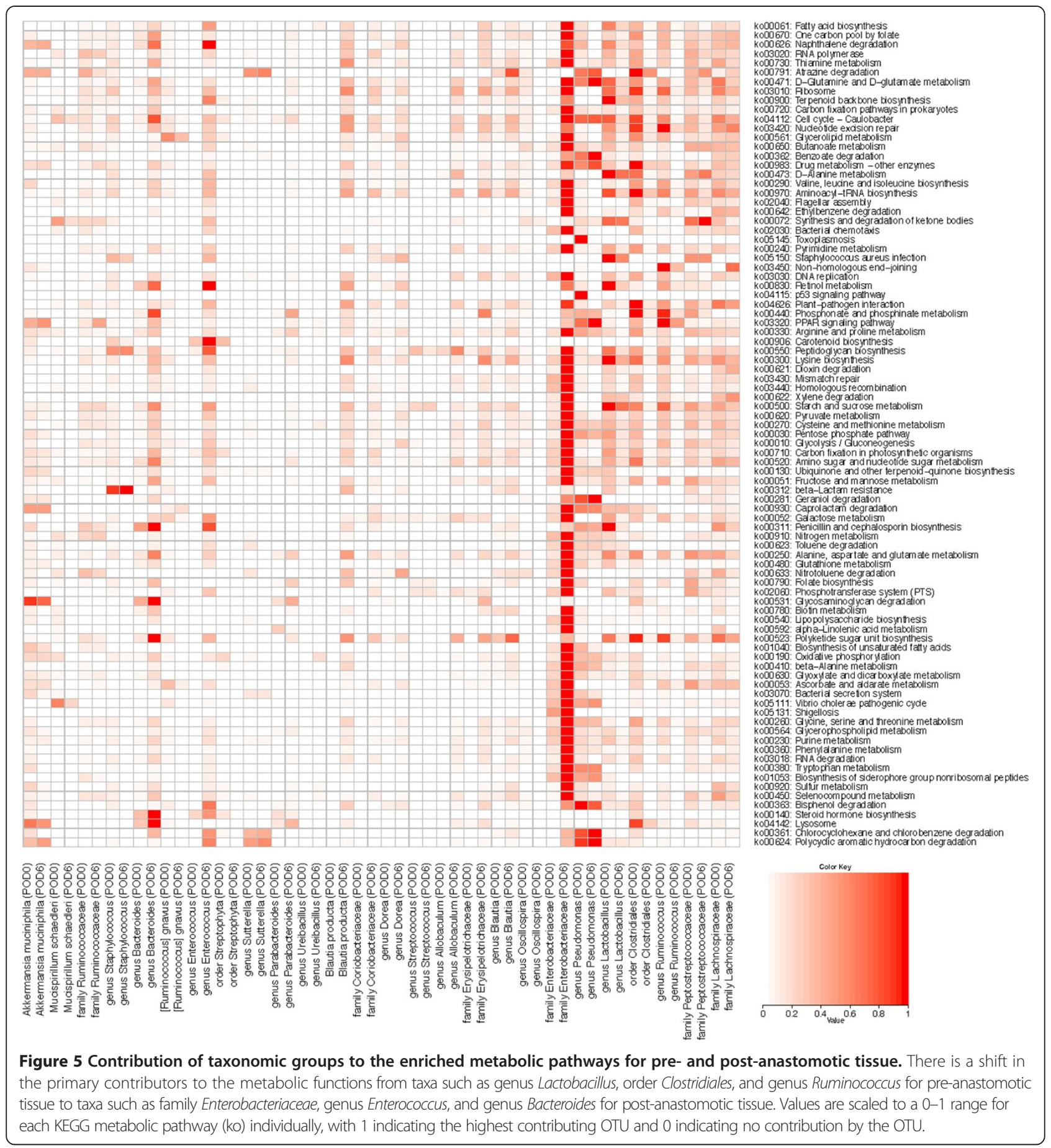

\section{S rRNA analysis of bacterial composition}

The following are the four groups of samples in our studies: (1) POD0 tissue for which the resected piece of colon was washed with saline and then swabbed for tissue associated microbiota; (2) POD0 luminal bacteria representing luminal content from a resected piece of colon; (3) POD6 tissue for which tissues at the site of anastomosis were collected, washed with saline, and then swabbed; (4) POD6 luminal bacteria representing luminal content from collected piece of anastomotic site of the colon. Twenty rats were included in the experiment. Samples with sequences containing low confidence base calls (phred $<20$ ) were eliminated. Samples were analyzed by $16 \mathrm{~S}$ rRNA V4 iTAG amplicon sequencing analysis. Following joining of paired $2 \times 150 \mathrm{bp}$ MiSeq read sequences (Illumina, San Diego, CA, USA) containing 
low confidence base calls (phred $<20$ ) were dropped, and the remaining sequences clustered into $97 \%$ similarity operational taxonomic units (OTUs) using uclust $[42,43]$. After dropping clusters composed of a single sequence, OTUs were assigned taxonomic annotations by aligning representative members of each cluster to the greengenes 12_04 database using the RDP classifier $[44,45]$. Sequencing depth among samples was normalized by rarefaction to a depth of 3,000 sequences per sample. Along with clustering, taxonomic assignment, and rarefaction, PCoA plots were derived from weighted unifrac distance matrices using the QIIME v1.6.0 software suite [46]. Significant differences in the abundance of taxonomic groups between treatments were calculated with Student's $t$ test followed by the Benjamini-Hochberg correction for multiple comparisons using an alpha of 0.05 [47]. Taxa tree was generated using GraPhlAn.

\section{Functional analysis of bacterial composition}

We predicted the functional composition and abundance of the microbial community using PICRUSt on the OTUs derived from the 16S rRNA analysis [6]. Differential functional abundance between postoperative day 0 and day 6 for stool contents and anastomosis tissue was performed using the Wilcoxon rank sum statistical test, and $p$ values were Bonferroni corrected for multiple testing. The metagenomic predictions produced by PICRUSt were further analyzed by HUMAnN [48] to provide KEGG metabolic pathway coverage, and LEfSe [49] was used for comparative analysis of the differentially enriched KEGG metabolic pathways between postoperative day 0 and day 6 for stool contents and anastomosis tissue samples. OTU contribution for enriched metabolic pathways was calculated by summing the abundance contribution of each OTU for each KEGG orthology comprising a metabolic pathway, and then normalizing by the number of samples. As there are hundreds of OTUs, a threshold was used to select just the top contributing OTUs for inclusion in Figure 5. The values were scaled to a $0-1$ range for each individual metabolic pathway, with 1 indicating the highest contributing OTU and 0 indicating no contribution by that OTU.

\section{Abbreviations}

POD: postoperative day; KEGG: Kyoto Encyclopedia of Genes and Genomes (a database resource for understanding high-level functions and utilities of the biological system); PICRUSt: Phylogenetic Investigation of Communities by Reconstruction of Unobserved States (a bioinformatics software package designed to predict metagenome functional content from marker gene (e.g.. $16 \mathrm{~S}$ rRNA) surveys and full genomes); OTUs: operational taxonomic units.
}

\section{Competing interests}

The authors declare that they have no competing interests.

\section{Authors' contributions}

BDS has made substantial contributions to conception and design and acquisition of data. DPS, SC, and JAG have made substantial contributions to data analysis and interpretation of data. OZ and JCA have made substantial contributions to conception, design, interpretation of data, and writing the manuscript. All authors have been involved in drafting the manuscript. All authors read and approved the final manuscript.

\section{Authors' information}

Jack A Gilbert, Olga Zaborina, and John C Alverdy are senior co-authors.

\section{Acknowledgements}

This study was funded by NIH RO1 2R01GM062344-13A1 (JCA). This work was supported in part by the U.S. Dept. of Energy under Contract DE-AC02-06CH11357.

\section{Author details}

'Department of Surgery, University of Chicago, 5841 S. Maryland, Chicago, IL 60637, USA. ${ }^{2}$ Institute for Genomic and Systems Biology, Argonne National Laboratory, 9700 South Cass Avenue, Argonne, IL 60439, USA ${ }^{3}$ Department of Ecology and Evolution, University of Chicago, Chicago, IL 60637, USA. ${ }^{4}$ Current address: Center for Metagenomics and Microbiome Research, Baylor College of Medicine, Houston, TX 77030, USA.

Received: 23 May 2014 Accepted: 4 August 2014

Published: 15 September 2014

\section{References}

1. Nelson RL, Gladman E, Barbateskovic M: Antimicrobial prophylaxis for colorectal surgery. Cochrane Database Syst Rev 2014, 5:CD001181.

2. Cohn I Jr, Rives JD: Antibiotic protection of colon anastomoses. Ann Surg 1955, 141(5):707-717.

3. Olivas AD, Shogan BD, Valuckaite V, Zaborin A, Belogortseva N, Musch M, Meyer F, Trimble WL, An G, Gilbert J, Zaborina O, Alverdy JC: Intestinal tissues induce an SNP mutation in Pseudomonas aeruginosa that enhances its virulence: possible role in anastomotic leak. PLoS One 2012, 7(8):e44326.

4. Wang Y, Antonopoulos DA, Zhu X, Harrell L, Hanan I, Alverdy JC, Meyer F, Musch MW, Young VB, Chang EB: Laser capture microdissection and metagenomic analysis of intact mucosa-associated microbial communities of human colon. Appl Microbiol Biotechnol 2010, 88(6):1333-1342.

5. Nava GM, Friedrichsen HJ, Stappenbeck TS: Spatial organization of intestinal microbiota in the mouse ascending colon. ISME J 2011, 5(4):627-638.

6. Langille MG, Zaneveld J, Caporaso JG, McDonald D, Knights D, Reyes JA, Clemente JC, Burkepile DE, Vega Thurber RL, Knight R, Beiko RG, Huttenhower C: Predictive functional profiling of microbial communities using 16S rRNA marker gene sequences. Nat Biotechnol 2013, 31(9):814-821.

7. Wallace AJ, Stillman TJ, Atkins A, Jamieson SJ, Bullough PA, Green J, Artymiuk PJ: E. coli hemolysin E (HlyE, ClyA, SheA): X-ray crystal structure of the toxin and observation of membrane pores by electron microscopy. Cell 2000, 100(2):265-276.

8. Sherlock O, Schembri MA, Reisner A, Klemm P: Novel roles for the AIDA adhesin from diarrheagenic Escherichia coli: cell aggregation and biofilm formation. J Bacterio/ 2004, 186(23):8058-8065.

9. Smith $J$, Bayles DO: The contribution of cytolethal distending toxin to bacterial pathogenesis. Crit Rev Microbiol 2006, 32(4):227-248.

10. Falzano L, Filippini P, Travaglione S, Miraglia AG, Fabbri A, Fiorentini C: Escherichia coli cytotoxic necrotizing factor 1 blocks cell cycle G2/M transition in uroepithelial cells. Infect Immun 2006, 74(7):3765-3772.

11. Korea CG, Badouraly R, Prevost MC, Ghigo JM, Beloin C: Escherichia coli K12 possesses multiple cryptic but functional chaperone-usher fimbriae with distinct surface specificities. Environ Microbiol 2010, 12(7):1957-1977.

12. Tran Van Nhieu G, Bourdet-Sicard R, Dumenil G, Blocker A, Sansonetti PJ: Bacterial signals and cell responses during Shigella entry into epithelial cells. Cell Microbiol 2000, 2(3):187-193.

13. Waters CM, Antiporta MH, Murray BE, Dunny GM: Role of the Enterococcus faecalis GelE protease in determination of cellular chain length, supernatant pheromone levels, and degradation of fibrin and misfolded surface proteins. J Bacteriol 2003, 185(12):3613-3623.

14. Park SY, Shin YP, Kim CH, Park HJ, Seong YS, Kim BS, Seo SJ, Lee $\mid H$ : Immune evasion of Enterococcus faecalis by an extracellular gelatinase that cleaves C3 and iC3b. J Immunol 2008, 181(9):6328-6336.

15. Boiko W, Krivorot'ko IV, Peev SB, Dotsenko EG, Lazirskii VA: Role of collagen in intestinal anastomosis formation after the rectum resection. Klin Khir 2009, 6:13-19. 
16. Brasken $\mathrm{P}$, Lehto $\mathrm{M}$, Renvall S: Fibronectin, laminin, and collagen types I, III, IV and V in the healing rat colon anastomosis. Ann Chir Gynaecol 1990, 79(2):65-71.

17. Hernandez LD, Pypaert M, Flavell RA, Galan JE: A Salmonella protein causes macrophage cell death by inducing autophagy. J Cell Biol 2003, 163(5):1123-1131

18. Walter J, Ley R: The human gut microbiome: ecology and recent evolutionary changes. Annu Rev Microbiol 2011, 65:411-429.

19. LeVeen HH, Wapnick S, Falk G, Olivas O, Bhat D, Gaurdre M, Patel M: Effects of prophylactic antibiotics on colonic healing. Am J Surg 1976, 131(1):47-53.

20. Mastboom WJ, Hendriks T, de Boer HH: Collagen changes around intestinal anastomoses in germ-free rats. Br J Surg 1989, 76(8):797-801.

21. Schardey HM, Kramling HJ, Cramer C, Kusenack U, Hadersbeck J, Schildberg FW: Risk factors and pathogenic microorganisms in patients with insufficient esophagojejunostomy after gastrectomy. Zentralb/ Chir 1998, 123(1):46-52.

22. Babrowski T, Holbrook C, Moss J, Gottlieb L, Valuckaite V, Zaborin A, Poroyko V, Liu DC, Zaborina O, Alverdy JC: Pseudomonas aeruginosa virulence expression is directly activated by morphine and is capable of causing lethal gut-derived sepsis in mice during chronic morphine administration. Ann Surg 2012, 255(2):386-393.

23. Babrowski T, Romanowski K, Fink D, Kim M, Gopalakrishnan V, Zaborina O, Alverdy JC: The intestinal environment of surgical injury transforms Pseudomonas aeruginosa into a discrete hypervirulent morphotype capable of causing lethal peritonitis. Surgery 2013, 153(1):36-43.

24. Shimizu K, Ogura H, Goto M, Asahara T, Nomoto K, Morotomi M, Yoshiya K, Matsushima A, Sumi Y, Kuwagata Y, Tanaka H, Shimazu T, Sugimoto H: Altered gut flora and environment in patients with severe SIRS. J Trauma 2006, 60(1):126-133.

25. Ohigashi S, Sudo K, Kobayashi D, Takahashi T, Nomoto K, Onodera H: Significant changes in the intestinal environment after surgery in patients with colorectal cancer. J Gastrointest Surg 2013, 17(9):1657-1664.

26. Clowes GH Jr, George BC, Villee CA Jr, Saravis CA: Muscle proteolysis induced by a circulating peptide in patients with sepsis or trauma. 1983. Nutrition 1995, 11(6):775. discussion 774; 776-777.

27. Kang CY, Halabi WJ, Chaudhry OO, Nguyen V, Pigazzi A, Carmichael JC, Mills S, Stamos MJ: Risk factors for anastomotic leakage after anterior resection for rectal cancer. JAMA Surg 2013, 148(1):65-71.

28. Lebreton F, Willems RJL, Gilmore MS: Enterococcus diversity, origins in nature, and gut colonization. In Enterococci: From Commensals to Leading Causes of Drug Resistant Infection. Edited by Gilmore MS, Clewell DB, Ike Y, Shankar N. Boston: Massachusetts Eye and Ear Infirmary; 2014.

29. Kaffarnik MF, Urban M, Hopt UT, Utzolino S: Impact of enterococcus on immunocompetent and immunosuppressed patients with perforation of the small or large bowel. Technol Health Care 2012, 20(1):37-48.

30. Komen N, Slieker J, Willemsen P, Mannaerts G, Pattyn P, Karsten T, de Wilt H, van der Harst E, van Leeuwen W, Decaestecker C, Jeekel H, Lange JF: Polymerase chain reaction for Enterococcus faecalis in drain fluid: the first screening test for symptomatic colorectal anastomotic leakage. The Appeal-study: analysis of parameters predictive for evident anastomotic leakage. Int J Color Dis 2014, 29(1):15-21.

31. Tomita H, Ike Y: Tissue-specific adherent Enterococcus faecalis strains that show highly efficient adhesion to human bladder carcinoma T24 cells also adhere to extracellular matrix proteins. Infect Immun 2004, 72(10):5877-5885.

32. Singh KV, Nallapareddy SR, Sillanpaa J, Murray BE: Importance of the collagen adhesin ace in pathogenesis and protection against Enterococcus faecalis experimental endocarditis. PLoS Pathog 2010, 6(1):e1000716.

33. Nallapareddy SR, Qin X, Weinstock GM, Hook M, Murray BE: Enterococcus faecalis adhesin, ace, mediates attachment to extracellular matrix proteins collagen type IV and laminin as well as collagen type I. Infect Immun 2000, 68(9):5218-5224.

34. Makinen PL, Clewell DB, An F, Makinen KK: Purification and substrate specificity of a strongly hydrophobic extracellular metalloendopeptidase ("gelatinase") from Streptococcus faecalis (strain 0G1-10). J Biol Chem 1989, 264(6):3325-3334.

35. Croxen MA, Finlay BB: Molecular mechanisms of Escherichia coli pathogenicity. Nat Rev Microbiol 2010, 8(1):26-38.

36. Ubeda C, Bucci V, Caballero S, Djukovic A, Toussaint NC, Equinda M, Lipuma L, Ling L, Gobourne A, No D, Taur Y, Jeng RR, van den Brink MR, Xavier JB, Pamer EG: Intestinal microbiota containing Barnesiella species cures vancomycin-resistant Enterococcus faecium colonization. Infect Immun 2013, 81(3):965-973.

37. Buffie CG, Pamer EG: Microbiota-mediated colonization resistance against intestinal pathogens. Nat Rev Immunol 2013, 13(11):790-801.

38. Sekirov I, Finlay BB: The role of the intestinal microbiota in enteric infection. J Physiol 2009, 587(Pt 17):4159-4167.

39. Mennigen R, Nolte K, Rijcken E, Utech M, Loeffler B, Senninger N, Bruewer M: Probiotic mixture VSL\#3 protects the epithelial barrier by maintaining tight junction protein expression and preventing apoptosis in a murine model of colitis. Am J Physiol Gastrointest Liver Physiol 2009, 296(5):G1140-G1149.

40. Mack DR, Ahrne S, Hyde L, Wei S, Hollingsworth MA: Extracellular MUC3 mucin secretion follows adherence of Lactobacillus strains to intestinal epithelial cells in vitro. Gut 2003, 52(6):827-833.

41. Okada M, Bothin C, Kanazawa K, Midtvedt T: Experimental study of the influence of intestinal flora on the healing of intestinal anastomoses. $\mathrm{Br} J$ Surg 1999, 86(7):961-965.

42. Aronesty $\mathrm{E}$ : Command-line tools for processing biological sequencing data. ea-utils. 2011. http://code.google.com/p/ea-utils.

43. Edgar RC: Search and clustering orders of magnitude faster than BLAST. Bioinformatics 2010, 26(19):2460-2461.

44. DeSantis TZ, Hugenholtz P, Larsen N, Rojas M, Brodie EL, Keller K, Huber T, Dalevi D, Hu P, Andersen GL: Greengenes, a chimera-checked 16S rRNA gene database and workbench compatible with ARB. Appl Environ Microbiol 2006, 72(7):5069-5072

45. Wang Q, Garrity GM, Tiedje JM, Cole JR: Naive Bayesian classifier for rapid assignment of rRNA sequences into the new bacterial taxonomy. Appl Environ Microbiol 2007, 73(16):5261-5267.

46. Caporaso JG, Kuczynski J, Stombaugh J, Bittinger K, Bushman FD, Costello EK, Fierer N, Peña AG, Goodrich JK, Gordon Jl, Huttley GA, Kelley ST, Knights D, Koenig JE, Ley RE, Lozupone CA, McDonald D, Muegge BD, Pirrung M, Reeder J, Sevinsky JR, Turnbaugh PJ, Walters WA, Widmann J, Yatsunenko T, Zaneveld J, Knight R: QIIME allows analysis of high-throughput community sequencing data. Nat Methods 2010, 7(5):335-336.

47. BYH Y: Controlling the false discovery rate: a practical and powerful approach to multiple testing. J R Stat Soc 1995, 57(1):289-300.

48. Abubucker S, Segata N, Goll J, Schubert AM, Izard J, Cantarel BL, Rodriguez-Mueller B, Zucker J, Thiagarajan M, Henrissat B, White O, Kelley ST, Methé B, Schloss PD, Gevers D, Metreva M, Huttenhower C: Metabolic reconstruction for metagenomic data and its application to the human microbiome. PLoS Comput Biol 2012, 8(6):e1002358.

49. Segata N, Izard J, Waldron L, Gevers D, Miropolsky L, Garrett WS, Huttenhower C: Metagenomic biomarker discovery and explanation. Genome Biol 2011, 12(6):R60.

doi:10.1186/2049-2618-2-35

Cite this article as: Shogan et al:: Intestinal anastomotic injury alters spatially defined microbiome composition and function. Microbiome 2014 2:35

\section{Submit your next manuscript to BioMed Central and take full advantage of:}

- Convenient online submission

- Thorough peer review

- No space constraints or color figure charges

- Immediate publication on acceptance

- Inclusion in PubMed, CAS, Scopus and Google Scholar

- Research which is freely available for redistribution 